\title{
Field of lines. From architecture to art, and back
}

\author{
Diego Perez-Espitia \\ Perez Reiter | Colombia | dpereze@gmail.com
}

\begin{abstract}
From architecture to art, and back to architecture: this research interrogates the potential of computation to bridge between both disciplines. The endeavour is twofold, a body of drawings is produced through algorithms that translate cultural and natural events into thousands of lines. Further on, these drawings are used to delineate a procedure in order to give rise to experimental buildings. By means of applying organizational principles outlined in the notion of fields, and the use of non-deterministic algorithms, a method to generate architecture from the realm of the visual arts is explored.
\end{abstract}

Keywords: Algorithm; Drawing; Architecture; Generative; Art.

\section{INTRODUCCIÓN}

El trabajo visual presentado en este artículo busca explorar vínculos significativos entre la arquitectura y el dibujo, a través del uso de técnicas de diseño algorítmico. Se fundamenta en la convicción de que estas disciplinas, aun siendo autónomas y claramente demarcadas, se alimentan discursiva y técnicamente.

Esta investigación tiene dos objetivos. 1. Producir una obra visual a través de técnicas digitales y principios de pensamiento algorítmico. 2. Interrogar las formas en que el dibujo, enmarcado en la tradición de las artes plásticas, puede ser usado como campo de experimentación abstracta en la producción de arquitectura. Dentro de esta lógica, las relaciones paramétricas entre miles de líneas se convierten en el instrumento que pone a dialogar a la arquitectura con el arte, en el marco de los medios digitales.

La relevancia del trabajo consiste en promover el desarrollo de las técnicas digitales en el arte y la arquitectura de Iberoamérica; superando la percepción del dibujo como una herramienta de representación, y usándolo como un instrumento de búsqueda estética y de experimentación durante el acto creativo propio del proyectar en arquitectura.

Es importante anotar que el autor es arquitecto, ha trabajado en diversos campos creativos, pero su énfasis profesional es la arquitectura.

\section{METODOLOGÍA}

El desarrollo de esta investigación comprende 4 fases, las tres primeras se gestaron durante los últimos seis años, fueron desarrolladas al interior del estudio Perez Reiter, una colaboración multidisciplinaria entre Irmgard Reiter y Diego Perez-Espitia, y se enmarcan en el primer objetivo mencionado en la introducción. La cuarta fase (en curso) se refiere al segundo objetivo de esta investigación y hace parte del trabajo actual del autor.

\section{OBJETIVO 1}

Producir una obra visual a través de técnicas digitales y principios de pensamiento algorítmico.

\section{FASE 1}

\section{EXPLORACIONES INICIALES. DIBUJO COMO HERRAMIENTA DE REPRESENTACIÓN}

Dado el énfasis computacional en el proceso de diseño de diversos proyectos al interior del estudio Perez Reiter, continuamente hacemos uso de algoritmos para realizar innumerables estudios de alternativas para el diseño de proyectos arquitectónicos. En muchos casos esas alternativas son interrogadas a través de dibujos bidimensionales que hacen parte de los primeros momentos del proceso de diseño. Son bocetos que permiten pensar el proyecto y dilucidar sus principales características. Esta investigación surge de varios dibujos realizados con esta intención.

\section{FASE 2}

\section{RECONOCIMIENTO DE POTENCIAL ESTÉTICO Y ESCRITURA DE ALGORITMOS}

Los miembros del estudio reconocen el valor estético de las imágenes realizadas en la etapa anterior e inician una búsqueda por generar una obra visual independiente, que no se encuentre al servicio de los proyectos arquitectónicos. Esta búsqueda está fundamentada en principios de pensamiento computacional y en la aplicación de técnicas digitales de diseño algorítmico.

En cuanto a las técnicas digitales empleadas, se escribieron diversos algoritmos en el lenguaje de programación Python para ser usados al interior del programa de modelado tridimensional Rhinoceros. Un algoritmo es una secuencia de instrucciones que permite llegar a la solución de un problema. Los algoritmos escritos en esta investigación tienen como punto de partida unos valores de entrada, datos numéricos de diverso orden, cuyo origen depende de la serie de dibujos en los que van a ser empleados (ver siguiente capítulo). Estos datos son 


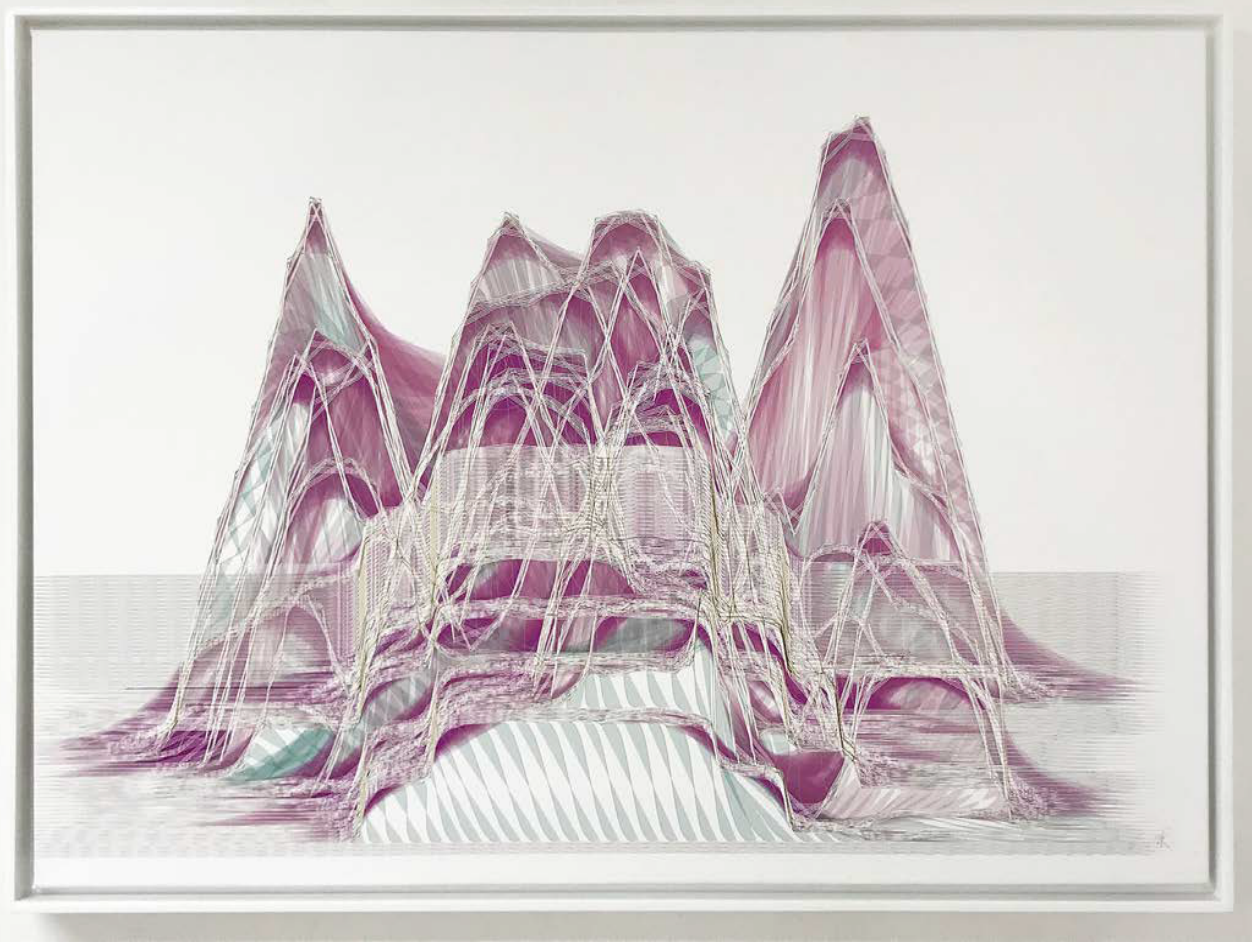

Figura 1: Beet 9th 17 01. Autor: Perez Reiter. Obra Resultado de la serie Música reescrita. El rango melódico y los picos de frecuencia de la introducción del tercer movimiento de la Novena Sinfonía de Beethoven es analizada y codificada en un algoritmo que genera este dibujo.

procesados por un sistema de condiciones matemáticas, un sistema de reglas que establece relaciones entre datos y procedimientos geométricos que, al ser ejecutado, manipula la dirección de cada línea que compone el dibujo, su relación con las líneas adyacentes y su longitud.

Los algoritmos desarrollados son no deterministas, sus resultados no son predecibles. Aun ingresando los mismos datos iniciales, la secuencia de instrucciones permite llegar a desenlaces muy diversos. Al mismo tiempo, tampoco es un algoritmo aleatorio. El sistema de reglas codificado está escrito de forma tal que la ejecución del algoritmo manifiesta comportamientos diversos, produciendo resultados que, aunque no son predecibles, se enmarcan dentro de la lógica interna del código; así, dentro del sistema de reglas establecido, son coherentes con la intención original del autor.

\section{FASE 3}

\section{CONSOLIDACIÓN DE OBRA VISUAL AUTÓNOMA}

El fundamento conceptual y la aplicación de las técnicas explicadas en la fase 2 de esta investigación, le permitieron al estudio producir un cuerpo de 72 piezas de diversos formatos, en lienzo quemado e impresión con tintas de pigmento en papel de algodón; expuesto en ferias, y exposiciones individuales y colectivas en galerías de arte de Bogotá, Londres, Nueva York, Los Ángeles, Pekín, Shanghái, Xiamen y Zúrich.

\section{OBJETIVO 2}

Interrogar las formas en que el dibujo, enmarcado en la tradición de las artes plásticas, puede ser usado como campo de experimentación abstracta en la producción de arquitectura.

\section{FASE 4 (EN CURSO)}

\section{IMPLEMENTACIÓN AL INTERIOR DE LA PRÁCTICA DE LA ARQUITECTURA}

Esta fase se refiere al trabajo en curso que se desencadena a partir de la escritura de este artículo. Una vez cumplidas las fases primera, segunda y tercera, el objetivo primordial de esta última fase es usar dichos dibujos para hacer edificios. En ese sentido, el autor los entiende como dibujos instrumentales. Dejando de lado aspectos puramente estéticos, que, aun siendo fértiles para el campo de la exploración de relaciones entre las disciplinas en juego durante este proceso, el arte y la arquitectura; en el contexto de esta conferencia, el autor considera más relevante enfocar este artículo en explorar dicha implementación desde el punto de vista de los procedimientos propios de la computación y las implicaciones de éstos en términos metodológicos y conceptuales. 


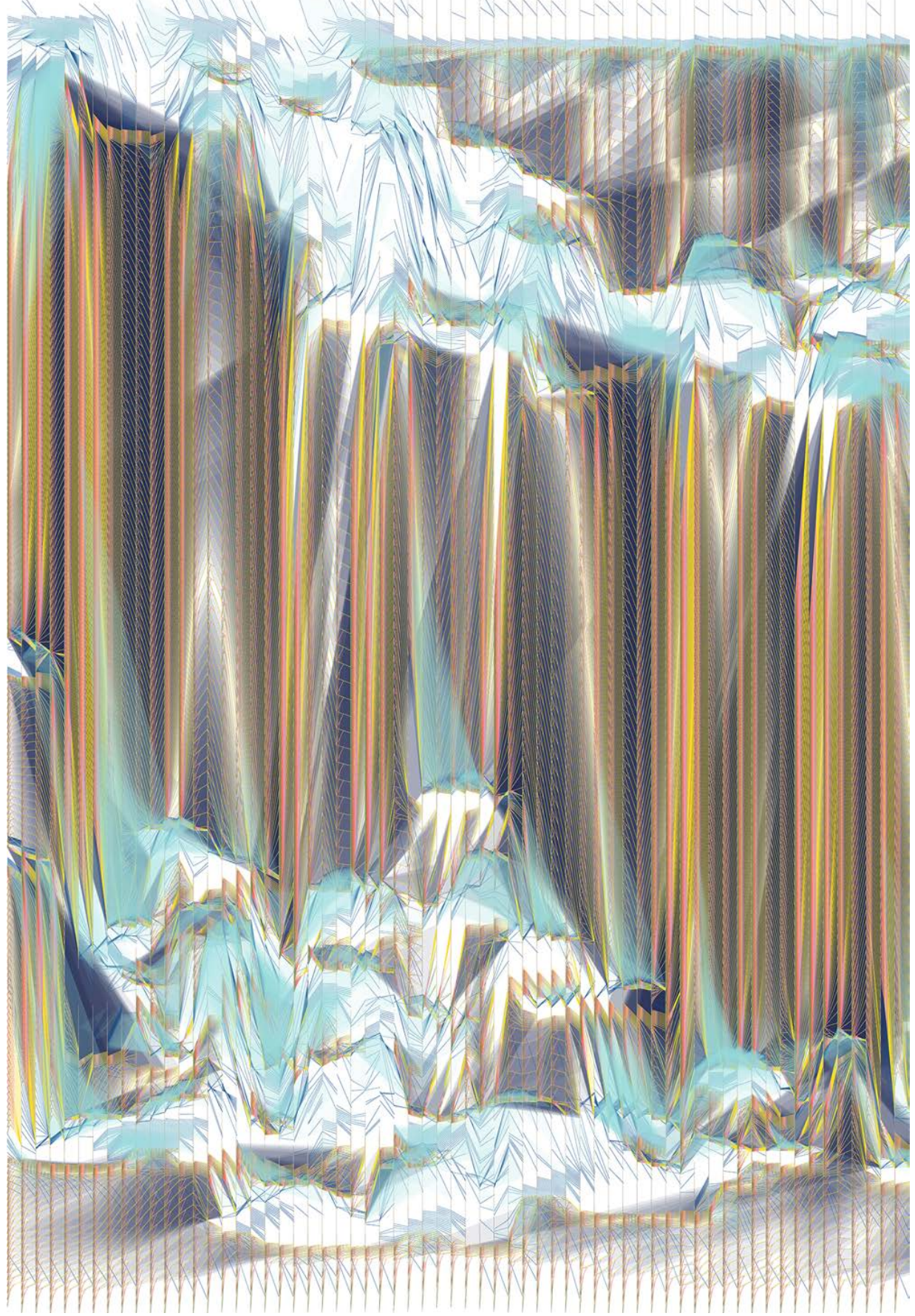

Figura 2: Quakes 37. Autor: Perez Reiter. Resultado de la serie Sismos reescritos. Datos de 5 años de sismos en la Costa Pacífica de América son codificados en un algoritmo para producir este dibujo. 


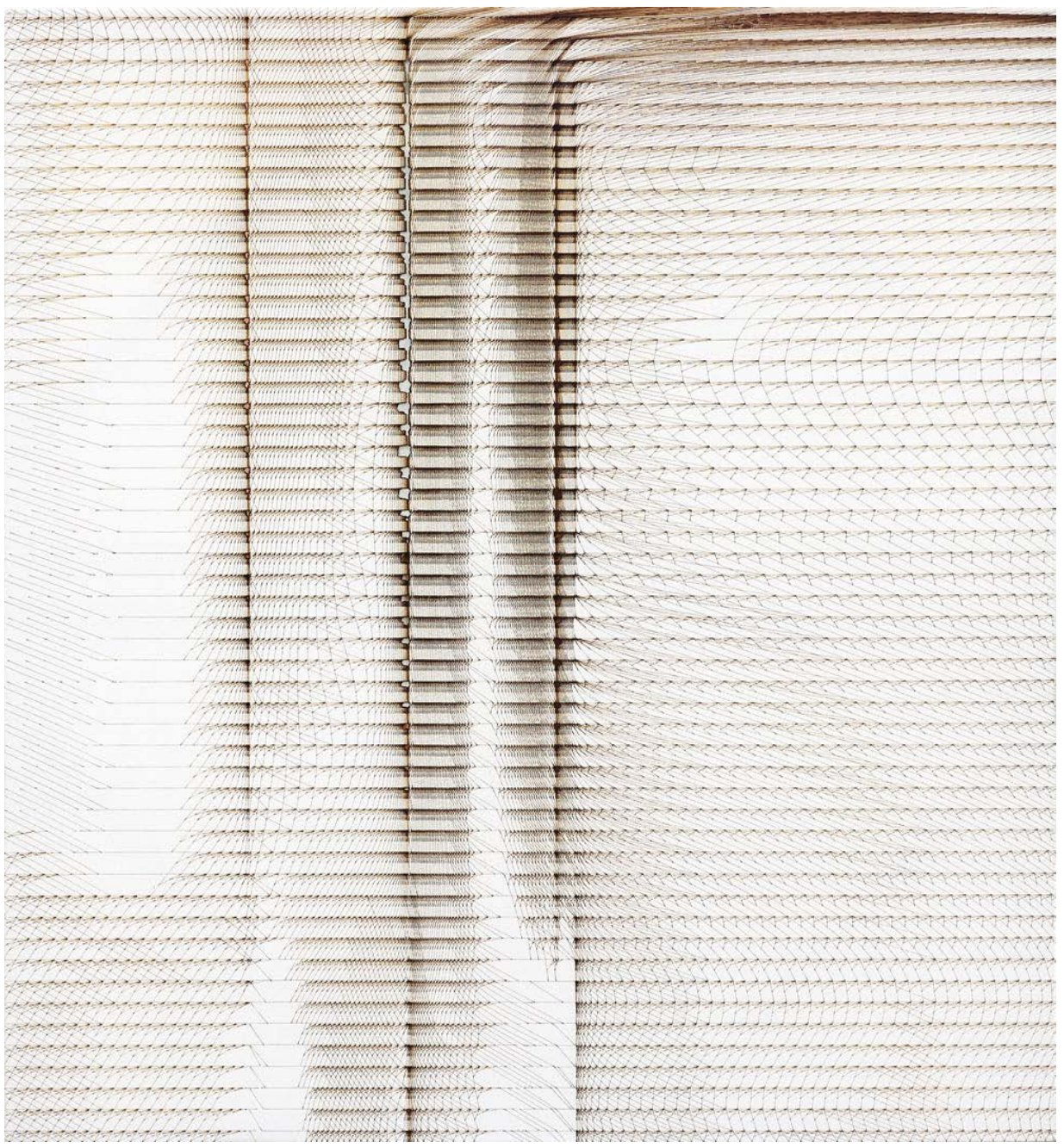

Figura 3: Quakes 31 x. Autor: Perez Reiter Dibujo resultado de la serie Sismos reescritos. La ejecución del mismo algoritmo usado para la figura 2 produce un resultado decididamente diferente.

\section{RESULTADOS}

\section{OBJETIVO 1}

Con respecto al primer objetivo de esta investigación, se ha producido una obra visual fundamentada en la escritura de algoritmos como acto creativo, los sistemas complejos, la manipulación de grandes cantidades de datos y el uso de técnicas de fabricación digital para la creación artística.

Estas obras interrogan la relación entre el espacio visual y el espacio construido a través de la computación, traducen eventos naturales y construcciones culturales en procedimientos algoritmicos Así, exploran las cualidades estéticas que surgen de la organización asociativa de miles de líneas. Desde los movimientos de la tierra bajo nuestros cuerpos, pasando por paisajes sonoros y la codificación de sistemas de notación, este trabajo tiene un profundo sentido de la espacialidad. Está compuesto por tres series temáticas:

Música reescrita: Estas piezas surgen de fragmentos de tres piezas de Beethoven que han sido analizados y codificados en un algoritmo. A través de un sistema de reglas, se dibuja música. Nuestros algoritmos transforman la continuidad de líneas paralelas en una compleja topografía de sensaciones. Crean paisajes sonoros basados en los picos de frecuencia y el rango melódico de las piezas, conjugando música, escritura y computación.

Sismos reescritos: Las obras producidas parten de un interés en estudiar cómo ciertos eventos naturales inciden en nuestra vida cotidiana. En ellas, la localización de los movimientos de placas tectónicas que ocurrieron en la Costa Pacífica de América, entre los años 2008 y 2013, ha 


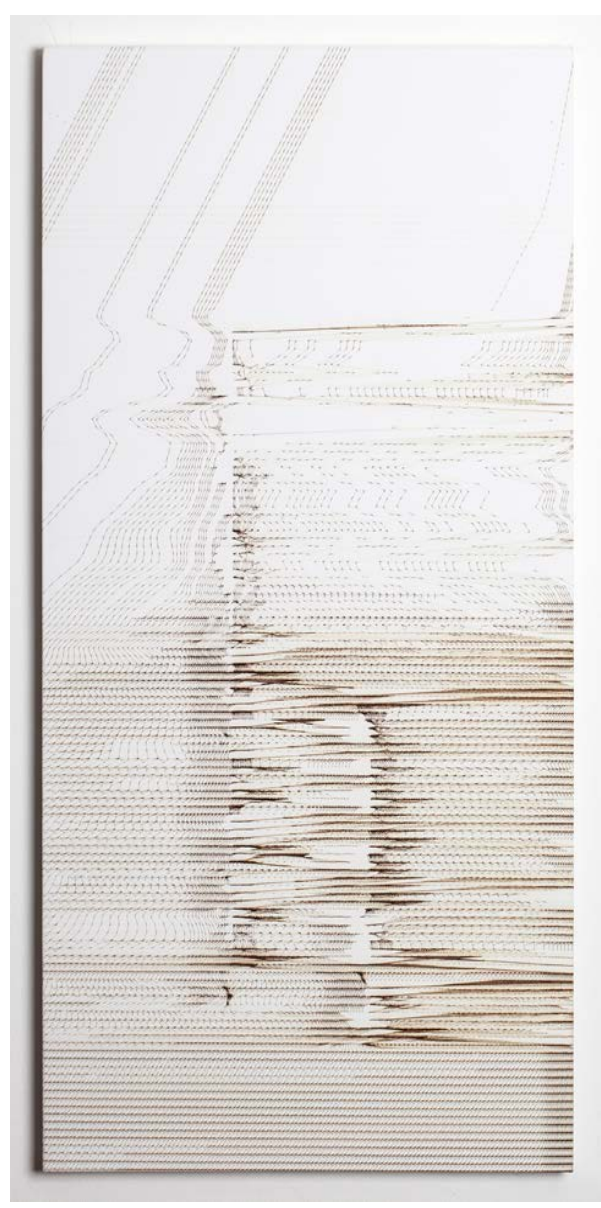

Figura 4: Lines written again. But who? Autor: Perez Reiter. Resultado de la serie Frases reescritas. La forma de las letras que componen frases específicas es analizada por un algoritmo para generar este dibujo.

sido codificada en un algoritmo escrito en el estudio del autor. Con la ayuda de datos rigurosamente administrados por el algoritmo, dibujamos miles de líneas que muestran cómo el tejido del día a día se rompe gracias a las fuerzas del sismo. La extraordinaria energía liberada a lo largo de esas 8 placas tectónicas se convierte en sutilezas sobre el lienzo. Sus interacciones se transforman en delgadas líneas sobre un fondo blanco. A medida que los cambios generados por el movimiento de la tierra debajo de nuestros cuerpos interrumpen el devenir continuo del espacio y del tiempo, la trama de lo cotidiano se expande, se comprime y se dobla.

Algunas de las cualidades de los sismogramas aparecen en estos dibujos, evocan su rigor científico. Al mismo tiempo, son impredecibles: los gráficos meticulosos, precisos y nítidos se filtran con un proceso estocástico, lo que permite que la representación de los datos codificados adquiera una nueva dimensión. Tal como en el dibujo a mano, la riqueza del trazo de una línea que surge al sostener un lápiz, se busca ahora mediante las particularidades de un proceso mecánico, tanto en la escritura del algoritmo como en el control modulado del proceso para quemar el lienzo.

Frases reescritas: Esta serie está fuertemente influenciada por el trabajo de Günther Uecker e Irma Blank. A partir de Uecker, desarrolla la noción de campo y de lógicas asociativas entre elementos, distanciándose de un acercamiento al elemento como objeto central de la obra. Fundamentado en la obra de Blank, referencia el acto de escribir, a través de un cuidadoso proceso creativo. El resultado visual emerge de la escritura de un algoritmo escrito por el autor, que analiza la forma de las letras que componen diferentes frases. Este análisis dispara una secuencia de instrucciones muy precisa que transforma la continuidad de las líneas que integran un patrón preestablecido.

Al ser estudiadas de cerca, estas piezas aluden a la pátina de los viejos manuscritos, el lienzo adquiere un color amarilloso en los puntos extremos de algunos trazos y en las intersecciones de varias líneas. La frase inicial esta ahora escondida tras un velo de procedimientos geométricos que emergen como un nido de miles de líneas.

\section{OBJETIVO 2}

Con respecto al segundo objetivo planteado en esta investigación. La escritura de este artículo ha permitido aclarar un marco de acción que define un camino de experimentación. Ésta busca implementar los desarrollos técnicos (escritura de código en el lenguaje de programación Python) y estéticos de los dibujos desarrollados, en proyectos de investigación en arquitectura.

En la actualidad hay diversos tipos de dibujo empleados en el desarrollo del proyecto arquitectónico, cada uno tiene una función específica. Los más relevantes responden a diferentes etapas del proceso de diseño. El boceto permite pensar el proyecto, los dibujos constructivos permiten comunicar al constructor las características (dimensiones, materiales, conexiones, articulaciones) de los elementos que componen el edificio. Los dibujos de presentación prefiguran el edificio y comunican cuáles son sus características espaciales, materiales, estructurales y organizacionales. Los dibujos objeto de esta investigación tienen una función diferente a las anteriores. Su propósito es estimular el proceso creativo y dirigirlo hacia ciertos preceptos más o menos abstractos. Estos preceptos son comunes al ejercicio profesional de la arquitectura y el arte, en el contexto de esta investigación tienen que ver con dos ámbitos específicos: lo organizacional y lo computacional. Es desde éstos que el marco de implementación se esboza en este trabajo.

Ya que el espacio común entre las dos disciplinas se refiere primordialmente a lo computacional y a lo organizativo, la manera de abordar dicha implementación es a través de un nuevo conjunto de algoritmos que permitan llevar los rasgos relevantes de los dibujos a un espacio donde cuestiones propias de la arquitectura puedan ser abordadas. Nos ocuparemos entonces de entender lo organizativo desde la técnica propia de los dibujos, el trazo 
de la línea, y así entender la relevancia de su utilización en arquitectura.

En el Renacimiento, la arquitectura se deslinda de la construcción como un acto de teoría y representación. En su tratado de 1452, Leon Battista Alberti lo declara:

"Comenzaremos, pues, así: Todo el negocio del edificar está constituido en lineamientos y fábrica, toda la fuerza y razón de los lineamientos se resume en que haya derecho y absoluto camino de componer y unir las líneas y los ángulos, con los cuales se comprenda y concluya la forma del edificio" (Alberti, 1747, p.8)

Desde ese momento, el delinear, trazar líneas, se convierte en el ingrediente primordial de la representación en arquitectura.

Por su parte, el arte ha tenido en el dibujo la herramienta universal para expresarse. En el marco de esta investigación, con el propósito de hacer un puente entre las dos disciplinas, la manera en que están dispuestos los miles de líneas que componen estos dibujos podría sugerir un camino simple a seguir: una línea se convertiría en un límite, en un muro. Una agregación de líneas construiría una superficie. Las líneas que sugieren un espacio en tres dimensiones se traducirían en un volumen. Si aplicamos los procedimientos compositivos de la modernidad en arquitectura esbozados en la década de 1920, y que prevalecen hasta hoy, ésta sería la manera obvia de pasar de una disciplina a otra. Abstraer y reemplazar. La traducción literal de lo que exhiben estos dibujos no tendría mayor interés en el ánimo por avanzar la aplicación de la computación en las artes y la arquitectura. Tal operación no se compadece con el cambio de paradigma que está gozando la arquitectura en las últimas décadas, y que ha sido explorado por la aplicación del pensamiento computacional en sus procesos creativos.

La implementación que propone esta investigación se hace a partir de preceptos que aúnan lo computacional con lo organizativo. Se hace así indispensable el uso de técnicas digitales de escritura de algoritmos para poder establecer las relaciones entre las líneas del dibujo. Lo algorítmico en el arte y la arquitectura habla primordialmente de las relaciones entre los elementos, más que de los elementos en sí mismos. Es la definición de estas relaciones lo que permite generar agregaciones de elementos donde las nociones modernas de composición se desvanecen y pierden relevancia. Ya no estamos hablando del todo y las partes, estamos hablando de la noción de campo, tal y como ha sido definida por Allen (2009), quien afirma que "El campo es una condición material, no una metáfora. Las condiciones de campo tienen que ver con la organización, con la materia y la fabricación" (p.158).

Adicionalmente, implementar los dibujos realizados en un proceso de diseño arquitectónico implica grandes grados de abstracción, ya que éstos carecen de escala, dimensiones, y materialidad. Tampoco se enmarcan en las representaciones típicas de la arquitectura: planta, sección, alzado, proyecciones isométricas y axonométricas.
"Quizá sea necesario un cambio más radical. Las condiciones de campo combaten los modos compositivos convencionales modernos del mismo modo que el movimiento moderno combatía las reglas clásicas de composición. La provisionalidad del todo debilita la aspiración clásica de totalidad, la similitud de las partes entre sí y la complejidad de la conexión opera contra la fragmentación moderna. En la condición de campo la forma global surge de las condiciones establecidas localmente." (Allen, 2009, p.167)

La noción de campo, entendido como un sistema de relaciones, donde no existe una definición explicita del dibujo -ni del edificio- sino la definición de las relaciones entre sus elementos (líneas en el caso de los dibujos, y elementos arquitectónicos en el caso de la arquitectura), constituye el núcleo de esta propuesta. El paso a seguir en esta investigación es diseñar un procedimiento algorítmico a partir del siguiente grupo de nociones comunes a lo computacional y a la idea de campo: En términos computacionales, sería necesario identificar patrones, reconocer tendencias, aplicar variabilidad, potenciar cambios en el tiempo, proponer lógicas asociativas y entender el proyecto arquitectónico como una secuencia de procedimientos. Dicho algoritmo debe también tener en cuenta asuntos organizacionales tales como cambios de densidades, concentraciones, intensidades, serialidad progresiva, relaciones locales entre elementos que generan un orden global, gradientes, diferenciación y acentuación.

\section{DISCUSIÓN}

A través de esta investigación y de la publicación de este artículo, se intenta alimentar el debate en torno a las diversas influencias que las disciplinas cobijadas por SIGRADI pueden ejercer entre sí, en particular, el arte y la arquitectura.

A partir de la autonomía de cada disciplina, en el marco de esta conferencia, aparece un vínculo factible y enriquecedor para establecer dichas relaciones. Lo algorítmico es uno de los vehículos para abordar cuestiones estéticas comunes a las dos disciplinas, permite abordar lo estético a partir del procedimiento, sacándolo del ámbito de la composición, y llevándolo al de lo generativo. Es a partir de éste que la arquitectura puede -nuevamente- ocupar el lugar de lo experimental, alimentándose del carácter disruptivo, cuestionador, y crítico del arte.

Por otro lado, tomando el camino más pragmático de esta reflexión, persiste una pregunta básica e ineludible. ¿Cómo tomar las nociones comunes a lo computacional y a la idea de campo, enumeradas en el capítulo anterior, y usarlas como puente entre el arte y la arquitectura? ¿Cómo pasar de la línea en el arte a la definición de un elemento arquitectónico sin que éste resulte siendo necesariamente la expresión de un límite? ¿Cuál es el potencial de lo computacional para lograr tal traducción entre disciplinas? 

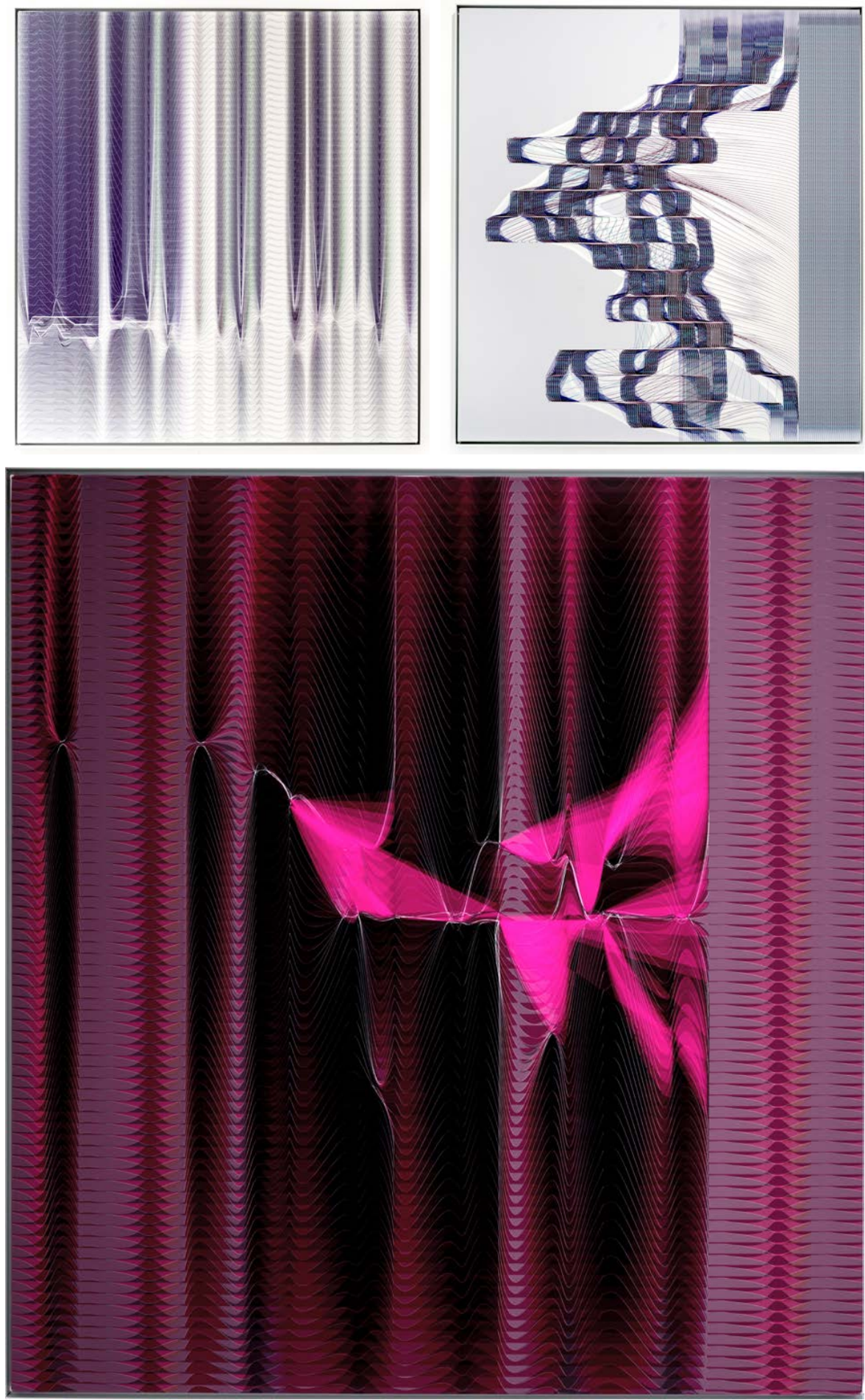

Figuras 5, 6 y 7: Beet LQ 127 3, Beet 9th 17 03, y Beet für Elise 03. Autor: Perez Reiter. Dibujos resultado de la serie Música reescrita. Tres piezas musicales diferentes son utilizadas como input para la la ejecución del mismo algoritmo, con resultados organizativos y estéticos diversos. 


\section{CONCLUSIÓN}

Este trabajo ejemplifica cómo a partir de un proceso de investigación que aplica principios y técnicas computacionales es posible establecer puentes entre el arte y la arquitectura. Aplicar en el arte posiciones teóricas del discurso contemporáneo en arquitectura permite producir un cuerpo de trabajo artístico que es posteriormente susceptible de alimentar una búsqueda en la arquitectura. En el curso de esta investigación, esto ha sido posible gracias a la versatilidad propia del trabajo con algoritmos, fundamentado en una posición clara frente a los modos de producción transdisciplinares.

"Da Vinci's sketches of machines leave us endlessly speculating about their foresightedness, Vladimir Tatlin is forgiven for designing an aeroplane that would never fly because it is more intriguing and more exploratory than many that do. All the time we must be ready to interrogate the architecture of the unlikely in order to push further the architecture of the predictable. Drawings and 'near-drawings' have a special role in all this." [Los bocetos de máquinas dibujados por Da Vinci nos hacen especular sin cesar sobre su previsión, Vladimir Tatlin es perdonado por diseñar un avión que nunca volaría porque es más intrigante y más exploratorio que muchos de los que lo hacen. Todo el tiempo debemos estar listos para interrogar la arquitectura de lo improbable y así impulsar aún más la arquitectura de lo predecible. Los dibujos y los "casi dibujos" tienen un papel especial en todo esto] (Cook, 2014, p.172).

\section{REFERENCIAS}

Alberti, L.B. (1747). Los diez libros de arquitectura. Tomo Primero. original: De re aedificatoria. Madrid, España: Joseph Franganillo

Allen, S (2009). Del objeto al campo: condiciones de campo en la arquitectura y el urbanismo en Abalos, I. (Ed.) Naturaleza y artificio. Barcelona, España: Gustavo Gili

Carrier, J., Neves, J. (Eds.) (2019). Irma Blank. Schrift wird zum bild. Köln, Alemania: König

Cook, P. (2014). Drawing. Chichester,Inglaterra: Wiley

Eckhard H., Krieger J. (Eds.) (2012). Günther Uecker: Word Pictures: Sixty Pictures in Four Decades. Berlín, Alemania: Jovis

Fry, Ch. (2019). Visuality and the haptic qualities of the line in generative art. XXII Generative Art Conference - GA2019. Recuperado

http://www.generativeart.com/GA2019_web/10_C_Fry_2_16 $8 \times 240 . p d f$

Kuddles, A., Marcus, A. (2018). Drawing codes. ACADIA // 2018:

Recalibration. On imprecision and infidelity. [Proceedings of the 38th Annual Conference of the Association for Computer Aided Design in Architecture. Recuperado de http://papers.cumincad.org/cgi-bin/works/Show?acadia18_46

Gleiginger, A., Vrachliotis, G. (2008). Complexity. Design strategy and world view. Basilea, Suiza: Birkhäuser Verlag AG

Hope, C., Ryan, J. (2014). Digital Arts: An Introduction to New Media. Londres, Inglaterra: Bloomsbury

Paul, Ch. (2015). Digital Art. Londres, Inglaterra: Thames and Hudson 